Article paru dans Critique internationale 11 (2001) , pp. 36-43

\title{
Falun Gong : la tentation du politique
}

David A. Palmer

La question du Falun Gong est le plus souvent vue en Europe à travers les deux prismes que sont la question des droits de l'homme de Chine et celle des sectes. Le Falun Gong a de quoi fasciner: a la fois «secte» et victime d'une répression sévère en Chine, il ne s'inscrit pas dans le schéma européen «sectes vs. démocratie », mais plutôt dans une nouvelle problématique de «secte vs. dictature». Comment se peut-il qu'un mouvement apocalyptique, bricolage du vieux fonds chinois de techniques de méditation et de pensée mystique, puisse devenir la principale force d`opposition en Chine, dépassant les dissidents démocrates en terms d'influence, en capacité d`organisation, en nombre d’adhérents et en tant que menace perçue par le pouvoir chinois? Le 25 avril 1999, le Falun Gong organisait à Pékin la plus grande manifestation ${ }^{1}$ vue en Chine depuis 1989. En janvier 2001, les journaux officiels chinois admettaient publiquement que malgré la sévère campagne de répression lancée par le pouvoir depuis l'été 1999, des adeptes du Falun Gong «ne cessent de se rendre à la place Tiananmen pour semer le désordre, d’y distribuer des bannières, des slogans et des tracts ... les méthodes de propagation employés par les éléments durs du «Falun Gong » ne cessent de se renouveler, ... ils se rendent sous des passerelles d’autoroutes de Pékin, dans des lieux publics très fréquentés et dans des quartiers résidentiels pour secrètement coller et distribuer des tracts illégaux et polluer l’atmosphère sociale.» Des milliers d'adeptes ont été incarcérés ou envoyés dans des camps de « rééducation par le travail »; une centaine de ces derniers seraient morts en détention.

\section{Une longue histoire de rébellions sectaires}

L'émergence de mouvements sectaires est un phénomène récurrent dans l'histoire religieuse de Chine. Il y a plus de 1800 ans, les révoltes millénaristes des sectes des Cinq Boisseaux de Riz et des Turbans Rouges furent liées à la naissance des premières communautés taoïstes. De la dynastie Yuan (14e siècle) jusqu'aux temps modernes, une constellation de réseaux annonce l'arrivée imminente de la fin apocalyptique du monde et l'inauguration d'un nouveau cycle universel par un 
nouveau Bouddha. Ces mouvements, dits du Lotus Blanc, employaient des techniques de respiration, de méditation et d'arts martiaux aujourd'hui appelées 'Qigong', pour recruter et former des communautés de pratiquants. Souvent liées à des rébellions politiques visant à renverser la dynstie régnante, les sectes du Lotus Blanc étaient sévèrement réprimées, les obligeant à devenir des sociétés secrètes. La dynastie mongole avait été renversée par une telle révolte, celle des Armées Rouges. C’est un officier de cette rébellion, Zhu Yuanzhang, qui fut le premier empereur de la dynastie des Ming -et qui, se retournant contre le milieu sectaire dont il était issu, promulgua des lois interdisant strictement l'hérésie, inaugurant une politique de suppression des mouvements religieux nouveaux et 'hérétiques' qui demeure en vigueur jusqu'à aujourd'hui. Les sectes hétérodoxes furent liées à une série de rébellions contre les dynasties Ming et Qing2; la rébellion de la secte des Taiping dans le milieu du 19e siècle affaiblit mortellement le régime impérial chinois. A la fin des Qing et durant la période républicaine (1911-1949), les sectes populaires se répandirent rapidement et connurent une influence grandissante, remplissant le vide laissé par la destruction des structures sociales traditionnelles et allant jusqu'à dominer l'organisation de villages et de régions entières. Le secret qui prévalait dans les sectes populaires depuis la répression accrue du 19e siècle se transforma même, dans quelques régions, en un recrutement public et massif. Par exemple, dans la période républicaine, la secte des Epées Rouges convertit des villages entiers de la Chine du nord avec le soutien des élites locales, qui trouvaient dans les pratiques et réseaux sectaires une méthode de résistance contre le banditisme et le pillage. Dès sa prise de pouvoir en 1949, le P.C. chinois lança une dure répression contre ces religions souterraines 'réactionnaires', menant à l'éradication presque totale du monde sectaire.

\section{Le rôle du Parti communiste dans la renaissance du monde sectaire}

Fondé en 1992, le Falun Gong est issu de la résurgence de ce monde sectaire dans les années 1980. En réalité, ce monde n'avait pas complètement disparu, mais s'était en partie reconstitué sous une forme nouvelle, derrière la vitrine des techniques traditionnelles de gymnastique respiratoire et méditative du 'Qigong'. Le Qigong avait suscité un immense engouement populaire dans les années 1980 et 90, largement grâce à l'encouragement et la protection de certains éléments du pouvoir central, de l'élite scientifique et des médias chinois. Ces derniers étaient persuadés que les pouvoirs

\footnotetext{
${ }^{1}$ Dix mille adeptes du mouvement s'étaient réunis autour de Zhongnanhai, centre névralgique du pouvoir central chinois, protestant l'arrestation de deux leaders de la secte arrêtés lors d'une autre manifestation contre un journal ayant publié un article critique sur le Falun Gong.

${ }_{2}^{2}$ Mentionnons à titre d'exemple les rébellions de Xu Hongru (1622), de Wang Lun (fin du 18e s.), et des Huit Trigrammes (1813).
} 
mentaux libérés par la méditation pourraient stimuler une renaissance de la civilisation chinoise et propulser la Chine à la tête d'une nouvelle révolution scientifique.

Cette étrange alliance entre le millénarisme communiste de la Chine Nouvelle et la mystique corporelle des maitres sectaires avait pris naissance avant même la fondation de la République populaire, lorsque des dirigeants du Parti dans la «zone libérée » de Yinan, privés de médecine moderne, ordonnaient une enquête de terrain sur les techniques populaires de gymnastique et de méditation. Ces méthodes, transmis dans les campagnes à travers des réseaux sectaires, furent alors purifiées de leurs éléments religieux, reformulées sous la nouvelle appelation de 'Qigong', et employées pour soigner des malades dans des cliniques pour cadres de l'Armée populaire de Libération.

Très vite, le Qigong se répandit dans les institutions médicales du nouvel état communiste. Dès 1953, un sanatorium spécialisé de Qigong était fondé à la villégiature de Beidaihe; l'élite du Parti s'initia à la méditation et aux techniques de relaxation. Liu Guizhen, le créateur du Qigong, fut honoré par Mao; de prestigieuses institutions médicales à Pékin et Shanghai ouvrirent des cliniques et des laboratoires de Qigong. Dans le contexte idéologique des années 1950 hostile à la médecine moderne «bourgeoise» et «occidentale», le Qigong, présenté comme une thérapie d'origine «populaire» et «chinoise», connut une première époque de floraison. Mais ce nouveau dressage politique ne suffit pas à protéger le Qigong contre la « Révolution culturelle » dirigée contre les cadres d'élite du Parti. Liu Guizhen et ses associés furent accusés de promouvoir des «superstitions féodales », et les institutions officielles du Qigong furent fermées au milieu des années 1960.

Durant la Révolution culturelle, le Qigong continua à être transmis dans des réseaux souterrains de maitres et de disciples. Dès le début des années 1970, Guo Lin, une femme guérie du cancer par la pratique du Qigong, osa cependant pratiquer et enseigner le Qigong en public, et inventa la pratique collective dans les parcs de Pékin - méthode qui permit la propagation rapide du Qigong auprès des masses. Guo Lin et sa méthode reçurent l'encouragement de plusieurs dirigeants du pouvoir central dès le commencement de la politique d'ouverture en 1979. Le Qigong était perçu par plusieurs dirigeants politiques comme une forme efficace de prévention et de thérapie facilement accessible aux masses, permettant d'augmenter le niveau de santé de la population sans nécessiter d'investissements publics.

En même temps, les médias chinois faisaient sensation autour de phénomènes étranges, tels que des enfants qui lisaient des caractères chinois avec leurs oreilles ou avec leurs aisselles, que des 
chercheurs prétendaient documenter et analyser de manière scientifique. Il apparut que des adeptes de Qigong pouvaient, par leurs pratiques méditatives, acquérir des pouvoirs de télékinèse, de voyance, de télépathie, etc. Les pouvoirs «extraordinaires» ou «surnaturels» du Qigong étaient même, parassait-il, prouvés par l'expérimentation scientifique. Par exemple, un chercheur d'une unité scientifique de haut niveau de Shanghai prétendit avoir détecté à l'aide d'un appareil, l'existence matérielle du «Qi externe» que l'adepte de Qigong peut cultiver mentalement et projeter de son corps pour guérir des malades à distance.

Dans la foulée de ces découvertes, le Qigong se répandit comme un feu de brousse à travers la Chine. Des guérisseurs traditionnels sortirent du placard, firent spectacle de leurs pouvoirs magiques maintenant légitimés comme 'fonctions exceptionnelles' à base scientifique, et se présentèrent comme "maitres de Qigong", enseignant des techniques de gymnastique et de méditation chinoises à des millions d'adeptes. Des milliers de maitres, d'écoles, d'associations, d'organisations de recherche et de publications voués au Qigong apparurent, expression d'une bouillonnante sous-culture reliant des dizaines de millions d'adeptes. Cette explosion du Qigong n'aurait pas été possible sans l'appui des enthousiastes du Qigong au sein de l'Etat, du Parti et du monde scientifique chinois, qui, dès 1982, obtinrent du Bureau central de Propagande l'interdiction de publication d'articles de presse critiquant les “fonctions exceptionnelles” du Qigong.

La Chine semblait prise d'un accès de "fièvre du Qigong". Cette gymnastique souvent simple et facile pouvait non seulement procurer santé et longévité à ses pratiquants, elle pouvait, selon ses adeptes, guérir les maladies, augmenter l'intelligence de l'homme, et lui donner des pouvoirs exceptionnels. L'expérience des adeptes et la recherche scientifique semblaient avoir prouvé la véracité des prouesses magiques des héros des légendes traditionnelles chinoises. La définition du "Qi externe" comme substance matérielle permettait d'étudier des phénomènes étranges et paranormaux dans le cadre légitime du matérialisme scientifique; l'hypothèse du Qigong comme moyen pratique de faire éclore des "fonctions exceptionnelles" latentes chez tous les hommes, laissait espérer que les pouvoirs mystiques jadis transmis de manière ésotérique à un nombre extrêmement restreint de personnes, étaient non seulement matériellement réels, mais pouvaient désormais, grâce à la science, être acquis par l'ensemble de la population. Le Qigong permettait de rêver à une démocratisation scientifique des traditions magiques chinoises. Le Qigong chinois était la clef permettant de se libérer des lois de la physique. Les vieux mythes étaient vrais, le Qigong allait les tranformer en science, et la Chine serait à l'avant-garde d'une nouvelle révolution scientifique mondiale. 
La "fièvre du Qigong” atteint son paroxysme en 1987 après la publication des résultats d'une expérience menée à l'université Qinghua, la plus prestigieuse faculté de sciences en Chine, qui affirmait que le 'Qi externe' émis par le maittre Yan Xin pouvait changer la structure moléculaire d'un échantillon d'eau à une distance de $2000 \mathrm{~km}^{3}$. Ce Yan Xin était à l'origine d'une nouvelle forme de Qigong, la 'conférence imbue de force' au cours de laquelle le maittre, tout en donnant une allocation sur la théorie scientifique du Qigong, émet du 'Qi externe' vers l'assistance. Les conférences de Yan Xin remplissaient des stades de dix à vingt mille personnes; une partie de l'auditoire tombait en transe et des malades et paraplégiques se trouvaient miraculeusement guéris.

L'euphorie fut de courte durée. La fulgurante popularité de Yan Xin rendit nerveux le pouvoir, qui organisa son exil aux Etats-Unis pour "promouvoir la culture chinoise" et "mener des expériences scientifiques". Les cas de psychose provoquée par une pratique abusive de la méditation se multiplièrent. L'escroquerie et le charlatanisme qui sévissaient au sein du monde du Qigong provoquèrent l'intervention de l'Etat, qui forma un comité de ministres chargé de la question du Qigong, et tenta de réglementer les associations de Qigong.

\section{La secte Zhong Gong}

L'Etat n'était pas seul à vouloir mettre de l'ordre dans le monde du Qigong. Le jeune maitre Zhang Hongbao tenta de récupérer les adeptes du Qigong sous le parapluie d'une vaste organisation commerciale possédant des universités de Qigong, des hôpitaux de Qigong, des instituts de gestion de cadres de Qigong, des entreprises de Qigong. Sa méthode du Zhong Gong intégrait les techniques de Qigong dans un système en huit stages ascendants. Dès le deuxième stage, les adeptes étaient intégrés dans le système de vente des cours d'apprentissage de la méthode, à travers une structure qui couvrait toute la Chine. En 1994, le Zhong Gong4 prétendait avoir 30 millions d'adeptes et constituait la plus grande organisation populaire en Chine en dehors du Parti communiste. Dès le milieu des années 1990, l'Etat commença discrètement à limiter les activités du Zhong Gong. Le gourou Zhang Hongbao disparut vers cette époque 5 .

Pour quelques années, le Zhong Gong avait réussi à dominer le monde du Qigong en intégrant les pratiques méditatives, respiratoires et de guérison à une structure bureaucraticocommerciale calquée sur celle du Parti communiste. Mais l'appétit du gain du maitre avait fait

\footnotetext{
${ }^{3}$ L'administration de l'université Qinghua dément tout lien avec ces expériences, qui auraient été organisées sans l'autorisation de l'université.

${ }^{4}$ Le nom Zhong Gong est un homophone du nom du Parti communiste chinois, Zhong Gong.
} 
contagion chez ses disciples; les ambitions personnelles et la corruption interne qui sévissaient au sein du Zhong Gong, avaient déjà considérablement affaibli la secte avant la répression de l’Etat.

\section{L'explosion du Falun Gong}

Un obscur maitre du nord-est chinois, Li Hongzhi, adopta une stratégie plus durable pour rallier le monde moribond du Qigong: associer les techniques corporelles et méditatives à une doctrine moraliste, messianiste et apocalyptique. Cette stratégie permit à sa méthode, le Falun Gong, de croître rapidement, alors même que, depuis 1995, les adversaires du Qigong dans le monde scientifique avaient provoqué une polémique dans la presse chinoise, dénonçant la ré-émergence de pratiques religieuses sectaires et de "superstitions féodales" sous le couvert du Qigong, ainsi que les expériences et recherches "pseudo-scientifiques” menées pour légitimer le Qigong.

Suite à cette polémique, le gouvernement lança en 1996 une campagne de "rectification" des associations de Qigong, enlevant la reconnaissance officielle aux écoles qui s'éloignaient trop du marxisme officiel. Dans ce climat moins favorable, de nombreux maitres de Qigong quittèrent la Chine pour l'Occident, intégrant le monde de la "médecine douce" et de l'orientalisme du "New Age". Le monde du Qigong se trouva éclaté et désorienté. Mais le Falun Gong, également victime de cette purge, continua son expansion, alors même que son chef Li Hongzhi se réfugiait aux EtatsUnis.

La tentative, encouragée le pouvoir, de récupérer les arts corporels, respiratoires et méditatifs au profit de la construction socialiste puis d'une révolution scientifique menée par la Chine, s'était donc soldée par un cuisant échec. Le Qigong avait donné lieu à l'apparition d'un monde de réseaux sectaires, d'organisations indépendantes de l'Etat dont la structure, l'idéologie, et les pratiques construisaient une société alternative dans les interstices de la société officielle. Avec le Falun Gong, l'évolution du Qigong dans une direction sectaire et quasi-religieuse atteignit son point culminant.

Les réseaux du Qigong laissaient entrevoir, dans leur structure, leur idéologie et leurs pratiques, une affinité avec les anciennes sectes hétérodoxes. Le Qigong est propagé par des organisations structurées qui emploient l'enseignement et la pratique de techniques corporelles et méditatives pour recruter des adeptes et transmettre une idéologie spécifique. Celle-ci, variant d'un réseau à un autre, propose typiquement un idéal de salut universel intégrant des éléments de pensée magique, d'eschatologie millénariste, de scientisme et de nationalisme romantique autour de la figure

\footnotetext{
${ }^{5}$ Il refit surface en été 2000, demandeur d'asile politique, sur l'île américaine de Guam dans le Pacifique.
} 
charismatique du Grand Maitre de Qigong. Ce modèle idéologique se propage subtilement dans un grand nombre d'organisations de Qigong; il se cristallise et apparait en plein jour dans le Falun Gong de Li Hongzhi ${ }^{6}$.

Le retournement de la communauté scientifique chinoise contre le Qigong en 1995 brisa le consensus politique et mèna à l'éclatement du monde du Qigong. Le Falun Gong se présenta non plus comme une méthode de Qigong mais comme une nouvelle Loi de l'Univers, transcendant toute forme d'organisation matérielle, supérieure à toutes les philosophies, lois, enseignements, religions, et méthodes de Qigong jamais apparues dans l'histoire de l'humanité, et offrant la seule voie de salut permettant de survivre à l'apocalypse devant bientôt détruire l'univers. La rigueur morale, le prosélytisme actif, et la stricte discipline de l'organisation du Falun Gong permirent à cette dernière de recruter largement dans tous les secteurs de la société, attirant des dixaines de millions d'adeptes, particulièrement parmi les retraités. Malgré le statut illégal et l'idéologie occultiste et anti-scientifique du mouvement, le Falun Gong se propagea ouvertement et en toute impunité. Seuls, quelques journalistes et scientifiques osèrent critiquer la secte dans les journaux, soulignant le caractère 'superstitieux' du mouvement et révélant des cas d'adeptes gravement malades ou morts pour avoir suivi l'enseignement de Li Hongzhi de refuser tout traitement médical.

Face à ces critiques, le Falun Gong réagit en organisant des manifestations devant les bureaux des médias concernés, exigeant (et obtenant parfois) des excuses publiques et la rétraction des articles offensifs. Malgré une telle cette audace - après tout, les médias chinois sont en quelque sorte les porte-paroles du gouvernement -- le pouvoir tarda à intervenir. Un grand nombre de membres du Parti communiste étaient adeptes ou sympathisants du Falun Gong; certains dirigeants considéraient la gymnastique quotidienne du Falun Gong comme un moyen économique et inoffensif d'occuper la masse désoeuvrée de personnes agées chinoises. D’autres redoutaient l'influence réelle du Falun Gong et craignaient de s'aliéner les dizaines de millions de disciples de Li Hongzhi.

\footnotetext{
${ }^{6}$ Parmi les caractéristiques du Falun Gong que l'on peut retrouver dans les anciennes sectes du Lotus Blanc, mentionnons les suivantes: (1) Doctrine de la fin imminente du monde ou du kalpa (cycle universel bouddhique); (2) Doctrine de l'arrivée imminente d'un nouveau Bouddha sauveur de l'humanité (Li Hongzhi va encore plus loin en se proclamant de loin supérieur au Bouddha). Notons en passant que les deux premiers caractères du nom de Li Hongzhi correspondent au nom du sauveur Li Hong attendu dans certaines traditions sectaires depuis des siècles. Le troisième caractère signifie "volonté", laissant entendre une 'volonté d'être le sauveur Li Hong". (3) Récitation collective de textes sacrés (le Zhuan Falun du Falun Gong) et pratique de techniques respiratoires et de méditation; (4) Réseau d'adeptes de constituant autour de la transmission de la doctrine et des techniques de méditation, se répandant sans limites géographies, sociales ou professionnelles; (5) rejet des institutions religieuses orthodoxes - le Falun Gong se proclame comme "Grande Loi" infiniment supérieure aux religions traditionnelles.
} 
Suite à l'arrestation de deux leaders d'une des manifestations du Falun Gong autour des bureaux d'un journal de Tianjin, la secte organisa le 25 avril 1999 une séance de méditation de plus de dix mille adeptes encerclant le Zhongnanhai, siège du pouvoir central à Pékin. Cet événement au puissant symbolisme, signifiant les visées politiques de la secte, provoqua l'ire du pouvoir qui décréta la suppression du Falun Gong le 23 juillet 1999. Dans les mois qui suivirent, la plupart des autres grandes organisations de Qigong furent discrètement démantelées par le gouvernement. Seul, le Falun Gong est devenu un mouvement de résistance souterrain qui continue sans relâche à narguer le pouvoir central, sous l'oeil des médias internationaux.

\section{Un vide spirituel et social}

Quels sont les implications et les résultats de la répression du Falun Gong? Selon un point de vue, le Falun Gong a été effectivement neutralisé; sa capacité de nuisance, aussi bien à l'intérieur du pays que dans les rapports extérieurs de la Chine, est un problème réel mais gérable. Mais une telle perspective néglige des problèmes de fond toujours loin d'être résolus, et dont l'affaire du Falun Gong n’est que le plus récent symptôme.

Premièrement, la persistance du fait religieux en Chine, démentant la confiance marxiste en une disparition graduelle de la religion grâce au développement économique et à l'abandon des structures sociales traditionnelles. En effet, en dessous du matérialisme extrême qui semble posséder la Chine actuelle, se profile une soif profonde de sens et de vie spirituelle. Pendant presque deux décennies suite à l'abandon du culte de Mao, le Qigong était devenu, dans les villes, la principale forme d'expression légitime de la religiosité populaire chinoise. Après la suppression du Falun Gong et le démantèlement des réseaux de Qigong, cette aspiration se trouve refoulée, à un moment où la 'crise de foi' (xinyang weiji) se ressent de manière aiguë.

Deuxièmement, la faiblesse de la société civile chinoise. Nous assistons depuis une dixaine d'années à l'émergence en Chine d'une sphère économique autonome face au politique. Mais le troisième pôle, celui de la société civile, peine toujours à trouver son autonomie. Le Qigong avait permis l'ouverture d'un espace relativement libre, dans laquelle purent foisonner une grande diversité de réseaux sociaux spontanés: groupes de pratiquants dans les parcs urbains, associations de chercheurs, revues et colloques, échanges internationaux, activités artistiques et écologiques, etc. Mais certains éléments du monde du Qigong, cherchant à commercialiser le Qigong, avaient miné la 
confiance des adeptes. Le Falun Gong, en s'opposant à cette tendance, avait réussi à rassembler les déçus de la corruption du monde du Qigong, et à constituer un noyau de fidèles prêts a sacrifier leur vie pour leur cause 7 . Mais la purge de 1996 et l'expansion fulgurante du mouvement en 1997-99 ont inexorablement mené le Falun Gong vers une logique de confrontation politique ${ }^{8}$.

L'espace de société civile créé par le monde du Qigong n'a donc pas réussi à trouver d'autonomie face aux logiques économique et politique. Le problème du Falun Gong met en relief la pauvreté du tissu social chinois. Cinquante ans de révolutions politiques puis d'obsession d'enrichissement économique ont donné un coup mortel aux structures sociales traditionnelles dans les villes, sans permettre la naissance de nouvelles formes stables de communauté. Hors de la famille nucléaire, du travail et de la consommation, il existe peu de cadres de vie associative en Chine urbaine. Ce vide social avait fourni un terrain fertile pour l'expansion des réseaux du Qigong puis du Falun Gong. Mais ces derniers, depuis la répression de 1999, sont réduits au statut de sociétés secrètes. Le vide social demeure: la Chine continue à souffrir de l'absence de société civile véritable.

David Palmer prépare une thèse à l'Ecole Pratique des Hautes Etudes, Section des sciences religieuses, sur l'histoire des réseaux de Qigong en Chine populaire.

Janvier 2001

\footnotetext{
${ }^{7}$ Certes, la vente de livres, de cassettes, etc. de Falun Gong, constitue une source de profits considérables pour Li Hongzhi et les éditeurs de ces produits. Mais les interactions au sein du Falun Gong (les formations et séances de pratique sont gratuites) sont perçus par les adeptes comme étant pures et libres de toute considération matérielle ou égoïste, contrairement à d'autres réseaux de Qigong et à la société en général). ${ }^{8}$ Le Falun Gong est souvent perçu à l'étranger comme rien de plus qu'un mélange de gymnastique chinoise et de spiritualité bouddhiste et taoïste. Or, dans un contexte totalitaire où le Parti communiste se veut comme l'unique mouvement de masse attirant dans son orbite toutes les autres grandes associations sociales, religieuses, etc., l'existence de toute autre organisation populaire de taille, qu'elle soit de nature politique ou non, ne peut manquer d'être perçue par tous les acteurs comme une menace réelle ou potentielle contre le monopole du Parti. Ceci déclenche non seulement une réaction défensive du Parti qui tentera de récupérer ou d'exterminer l'organisation rivale, mais peut aussi provoquer des ambitions politiques au sein de cette dernière. Li Hongzhi, conscient de son pouvoir sur des dizaines de millions d'adeptes chinois, s'est ainsi mis à orienter son influence vers le domaine politique. Les premières manifestations du Falun Gong en 1998 et 1999, contre des médias officiels chinois, signalent une puissance politique désireuse et capable de faire plier des organes d'Etat. Dans la grande manifestation de Zhongnanhai du 25 avril 1999, le public chinois fut témoin que le Falun Gong ne craignait même pas d'affronter le pouvoir suprême. Un adepte me disait à cette époque, d'un ton menançant: "si le gouvernement ose toucher au Falun Gong, Li Hongzhi montrera son pouvoir”.
} 
